\title{
Pressemitteilung zur organisatorischen und disziplinarischen Unterstellung der MTRA unter die Pflegedirektion
}

\begin{abstract}
Fachverbände: Unterstellung der MTRA unter Pflegedirektion rechtlich nicht vertretbar Der Dachverband für Technologen/-innen und Analytiker/-innen in der Medizin e. V. (DVTA), die Vereinigung Medizinisch -Technischer Berufe in der DRG (VMTB) und die Deutsche Röntgengesellschaft e. V. (DRG) sind sich einig: Die Pflegedirektion ist nicht befugt, fachliche Weisungen in der Radiologie zu erteilen. Neben den rechtlichen Aspekten geht es außerdem um Personalwirtschaft sowie um Haftungsfragen.
\end{abstract}

Berlin/Hamburg, März 2014. „Das MTAGesetz regelt es eindeutig“, so Elske Müller-Rawlins, Rechtsanwältin und Vorstandsreferentin des DVTA, „Medizinischtechnische Assistenten dürfen nur auf Anforderung eines Arztes die ihnen vorbehaltenen Tätigkeiten ausüben." In einer Stellungnahme des DVTA heißt es zudem weiter, dass selbst bei Vorhandensein eines akademischen Abschlusses der Pflegedienstleitung diese Unterstellung nicht korrekt ist, da die erforderlichen Fachkenntnisse, Fähigkeiten und Fertigkeiten nicht vorhanden sind. Diese sind aber dringend notwendig, um fachliche Anweisungen zu vorbehaltenen Tätigkeiten geben zu können. Gerade um Patienten im sensiblen Bereich der Radiologie optimal zu versorgen, muss die Verantwortung bei fachkundigen Ärzten liegen. Die Pflegedienstleitung darf den MTA daher weder fachliche Anordnungen geben, noch ist sie selbst in der Lage, die vorbehaltenen Tätigkeiten selbst auszuführen, d.h. sie benötigt ihrerseits eine ständige Aufsicht. Dies ist nicht nur aus fachlicher, sondern auch aus personalwirtschaftlicher Sicht nicht zielführend. Im Bereich der Radiologie kommt noch hinzu, dass MTRA über die Fachkunde im Strahlenschutz verfügen müssen, die ansonsten lediglich Radiologen und Medizinphysiker besitzen.
Selbst die alleinige Delegation der organisatorischen Abteilungsleitung über die MTRA auf die Pflegedirektion macht schon allein aus personalwirtschaftlichen Gründen wenig Sinn. Denn neben der (organisatorischen) Pflegedirektion wird auch gleichzeitig eine (fachlich) leitende MTRA benötigt, was zu einer Doppelspitze führen würde und damit zu höheren Personalkosten. Zudem bedarf es der genauen Beobachtung von Richtlinienänderungen der Bundesärztekammer, von Gesetzesänderungen, der Organisation von adäquaten Fortbildungsmaßnahmen wie bspw. Fachkundeaktualisierungskurse.

Ein 3. und bedeutender Punkt betrifft den Haftungsaspekt für die Klinikbetreiber. Denn diese laufen Gefahr in die organisatorische Haftung mit einbezogen zu werden, sollte sich ein Fehlverhalten der Pflegedienstleitung bei der Wahrnehmung von organisatorischen Aufgaben von MTA herausstellen. Sofern die Pflegedienstleitung - wie bei einer leitenden MTA üblich - auch vorbehaltene Tätigkeiten ausführt, um z.B. Personalengpässe mit auszugleichen, und es dabei zu einer Patientenschädigung kommt, ist auch hier die Haftung des Klinikbetreibers klar gegeben, insbesondere wenn die geforderte stete Auf-

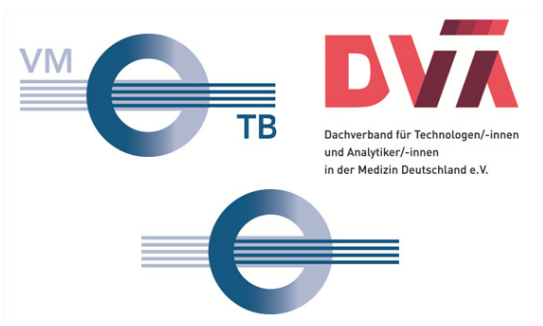

sicht durch einen fachkundigen Arzt nicht gewährleistet wurde.

Nach Auffassung des DVTA, der VMTB und der DRG ist es daher rechtlich nicht zulässig und personalwirtschaftlich nicht sinnvoll, die MTRA einer Röntgenabteilung, Nuklearmedizin und Strahlentherapie organisatorisch und dienstrechtlich der Pflegedirektion zu unterstellen.

\section{Pressekontakt}

Nina Keil

Pressereferentin

Deutsche Röntgengesellschaft e.V.

Tel.: 030-916 07025

Fax: 030-916 07022

E-Mail: keil@drg.de

Internet: www.drg.de www.vmtb.de 